\title{
EQUAÇÕES DE ESTIMATIVA DA EVAPOTRANSPIRAÇÃO DE REFERÊNCIA (ETO) PARA A REGIÃO DE BALSAS-MA
}

Jhonata Santos Santana ${ }^{1}$, Elton Ferreira Lima ${ }^{2}$, Wilson Araújo da Silva ${ }^{3}$, Mydilany Carneiro Fernandes ${ }^{4}$, Maria Ivanessa Duarte Ribeiro ${ }^{5}$

1. Bolsista de Apoio Técnico Institucional II (BATI II), Engenheiro Agrônomo, Universidade Estadual do Maranhão-UEMA. Imperatriz, MA. agro.starf@gmail.com

2. Bolsista de iniciação científica, Graduando em Engenharia Agronômica, Universidade Estadual da Região Tocantina do Maranhão-UEMASUL, Imperatriz, MA.

3. Doutor em Ciência do Solo, Engenheiro Agrônomo, Universidade Estadual do Maranhão, Imperatriz, MA.

4. Graduada em Ciências Biológicas na Universidade Estadual do Maranhão-UEMA, Imperatriz, MA.

5. Bolsista de iniciação científica, Graduando em Engenharia Agronômica-Universidade Estadual da Região Tocantina do Maranhão-UEMASUL, Imperatriz, MA.

Recebido em: 06/04/2018 - Aprovado em: 10/06/2018 - Publicado em: 20/06/2018 DOI: $10.18677 /$ EnciBio_2018A43

\begin{abstract}
A determinação da evapotranspiração é essencial para o manejo correto da água na agricultura, pois é o principal parâmetro empregado na estimativa de demanda hídrica das culturas que é a base para o adequado manejo dos sistemas de irrigação e, por isto, a escolha do modelo de estimativa deve ser extremamente criteriosa. Assim, objetivou-se neste estudo investigar o desempenho da ETo obtida por 10 diferentes metodologias em comparação ao método padrão de PenmanMonteith de FAO-56, para a localidade Balsas-MA, utilizando uma série histórica contínua de 18 anos $(2000$ - 2017) de dados climáticos. A avaliação de desempenho dos métodos foi baseada nos coeficientes de correlação " $r$ ", de concordância "d" de Willmott e o índice de desempenho "c". Além disso, os resultados obtidos pelos diferentes métodos foram correlacionados por meio de regressão linear tendo como padrão o método Penman-Monteith-FAO 56. Dentre os modelos testados o método de Turc (ETo TC) apresentou melhor índice de confiança 0,80 "Bom", apresentando maior confiabilidade na estimativa da evapotranspiração de referência. Por outro lado, os métodos de Linacre, BenevidesLopez, Radiação Global, Jensen-Haise, Camargo e Ivanov apresentaram os piores desempenhos em relação ao método de Penman-Monteith-FAO.
\end{abstract}

RESUMO

PALAVRAS-CHAVE: desempenho, métodos, Penman-Monteith-FAO

\section{ESTIMATIVE OF EQUATIONS OF THE REFERENCE EVAPOTRANSPIRATION (ETO) FOR THE REGION OF BALSAS-MA}

ABSTRACT 
The determination of evapotranspiration is essential for the correct management of water in agriculture, since it is the main parameter used in estimating the water demand of the crops that is the basis for the adequate management of irrigation systems and, therefore, the choice of the estimation method must be extremely careful. Thus, the objective of this study was to investigate the performance of ETo obtained by 10 different methodologies compared to the FAO-56 Penman-Monteith standard method for the Balsas-MA locality using a continuous historical series of 18 years (2000 - 2017 ) of climatic data. The performance evaluation of the methods was based on Willmott's correlation coefficients " $r$ ", concordance " $d$ " and performance index "c". In addition, the results obtained by the different methods were correlated by linear regression using the Penman-Monteith-FAO method 56. Among the models tested, the Turc (ETo TC) method presented a better confidence index 0.80 "Good ", Presenting greater reliability in the estimation of reference evapotranspiration. On the other hand, the methods of Linacre, Benevides-Lopez, Global Radiation, Jensen-Haise, Camargo and Ivanov presented the worst performances in relation to the Penman-Monteith-FAO method.

KEYWORDS: Performance, methods, Penman-Monteith-FAO

\section{INTRODUÇÃO}

O conhecimento dos valores de evapotranspiração é essencial para o planejamento na utilização dos recursos hídricos e manejo da água na agricultura irrigada (CUNHA et al., 2013). A determinação da demanda de hídricas das culturas, para fins de manejo da irrigação, pode ser feita a partir de medições de parâmetros que podem ser efetuadas no solo, na planta e na atmosfera (GIOVANELLI et al., 2015). Para a determinação do consumo de água das culturas a partir de medições atmosféricas foram desenvolvidos diversos modelos, que vão desde complexas equações agrometereológicas, alimentadas por um número considerável elementos climatólogicos (como o de Penman-Monteith) até modelos simplificados que se baseiam em apenas uma variável metereológica para estimar a evapotranspiração de referência (ETo) (RODRIGUES et al., 2013).

O modelo agrometeorológico considerado internacionalmente o mais preciso na estimativa da ETo é o Penman-Monteith/FAO-56, entretanto este apresenta restrições de utilização devido a exigência de muitos parâmetros meteorológicos para sua execução (PALARETTI et al., 2014). Segundo Carvalho et al. (2015) a utilização de modelos físico-matemáticos tem se tornado a alternativa mais prática para a estimação da ETo.

Assim, uma alternativa bastante utilizada quando as estações meteorológicas não fornecem os elementos climáticos necessários a aplicação do modelo de Penman-Monteith é a estimativa da ETo por outras equações existentes. Mas antes da escolha de um determinado método, é importante avaliar sua adaptabilidade ao clima de uma determinada região, verificando seu desempenho em relação ao modelo padrão de estimativa da ETo, o Penman-Monteith/FAO-56.

Deste modo o presente estudo propõe uma contribuição para o uso racional da água na agricultura irrigada para a localidade de Balsas/MA, um dos maiores produtores de grãos do MATOPIBA. O Matopiba refere-se ao espaço geográfico composto pelos estados do Maranhão, Tocantins, Piauí e Bahia, cujas siglas dão nome a essa região. Que a partir da publicação do Decreto $n^{\circ} 8.447$ de 2015, passou a ser foco de grandes investimentos de recursos públicos no âmbito do 
Plano de Desenvolvimento Agropecuário (PDA) do Matopiba (FERREIRA et al., 2017).

Efetivamente, o objetivo do presente estudo foi estimar a ETo através das equações de Turc, Makkink, Jensen-Haise, Camargo, Priestley-Taylor, Ivanov, Linacre, Hargreaves-Samani, Benevides-Lopez e Radiação Global afim de encontrar o modelo que estime satisfatoriamente a evapotranspiração de referência (ETo) em relação ao modelo padrão de Penman-Monteith/FAO-56 para Balsas-MA.

\section{MATERIAL E MÉTODOS}

O presente estudo foi desenvolvido para a região sul do Maranhão, mais precisamente para a região de Balsas, as coordenadas geográficas do município de Balsas são $07^{\circ} 31^{\prime} 57^{\prime \prime} \mathrm{S}$ de latitude $S$ e $46^{\circ} 02^{\prime} 08^{\prime \prime}$ de longitude W, com de altitude 237 metros acima do nível do mar. O clima de Balsas é classificado pela metodologia de Köpen como (Aw), tropical quente e úmido. Com temperatura média anual de $26,4^{\circ} \mathrm{C}$ e precipitação média anual de $1190 \mathrm{~mm}$. No quadro 1 estão descritas as principais características das equações de estimativa da ETo utilizadas neste estudo.

QUADRO 1- Modelos empregados na estimativa da evapotranspiração de referência (ETo).

\begin{tabular}{|c|c|c|}
\hline Método & $\begin{array}{l}\text { Parâmetros } \\
\text { meteorológicos } \\
\text { de entrada }\end{array}$ & Características \\
\hline $\begin{array}{l}\text { Penman- } \\
\text { Monteith/FAO } \\
\text { (PMF) }\end{array}$ & $\begin{array}{l}\text { Tmax }{ }^{*} \text { Tmed, Tmin, } \\
\text { URmed, } \\
\text { Vv, n, Pr, Rso, Rstop }\end{array}$ & $\begin{array}{l}\text { Conhecido também como Penman-Monteith } \\
\text { FAO 56, é considerado a equação } \\
\text { agrometeorológica mais precisa e apropriada } \\
\text { para cálculo da ETo. }\end{array}$ \\
\hline Turc (Tc) & Tmed, URmed, Rso & $\begin{array}{l}\text { Equação simples de estimativa da ETo que } \\
\text { utiliza valores de temperatura, umidade } \\
\text { relativa e radiação solar. }\end{array}$ \\
\hline $\begin{array}{l}\text { Heagreves e } \\
\text { Samani (HG) }\end{array}$ & Tmax, Tmed, Tmin & $\begin{array}{l}\text { Conhecido também como } \\
\text { "Hargreaves", um dos mais utilizados na } \\
\text { estimativa da ETo em regiões áridas. }\end{array}$ \\
\hline Makkink (MK) & $\begin{array}{l}\text { Pv, Tmax, Tmed, } \\
\text { Tmin,Rso }\end{array}$ & $\begin{array}{l}\text { Método holandês, um dos mais utilizados no } \\
\text { leste europeu na estimativa da ETo. }\end{array}$ \\
\hline Camargo (CM) & Tmed, Rstop & $\begin{array}{l}\text { Baseado em Thornthwait apresentando } \\
\text { desempenho semelhante. }\end{array}$ \\
\hline $\begin{array}{l}\text { Priestley \& Taylor } \\
\text { (PT) }\end{array}$ & $\begin{array}{l}\text { Rn, Tmax, Tmed, } \\
\text { Tmin }\end{array}$ & $\begin{array}{l}\text { Abordagem simples para estimar a ETo que } \\
\text { exige dados de declividade da curva de } \\
\text { pressão; saldo de radiação e fluxo de calor no } \\
\text { solo. }\end{array}$ \\
\hline Linacre (LN) & Tmed, Tpo & $\begin{array}{l}\text { Método baseado na altitude e latitude que } \\
\text { utiliza a temperatura média do ar e } \\
\text { temperatura do ponto de orvalho na estimativa } \\
\text { da ETo. }\end{array}$ \\
\hline Jensen-Haise (JH) & Rs, Tmed & $\begin{array}{l}\text { Método para régiões áridas e semiáridas que } \\
\text { utiliza temperatura média do ar e radiação } \\
\text { solar. }\end{array}$ \\
\hline Ivanov (IV) & Tmed, URmin & $\begin{array}{l}\text { Metodologia simples utilizado na estimativa da } \\
\text { ETo para períodos menores que } 30 \text { dias }\end{array}$ \\
\hline
\end{tabular}




\begin{tabular}{|c|c|l|}
\hline $\begin{array}{c}\text { Benevides Lopez } \\
\text { (BL) }\end{array}$ & Tmed & $\begin{array}{l}\text { Método que utiliza e temperatura média e } \\
\text { umidade relativa do ar na estimativa da ETo. }\end{array}$ \\
\hline $\begin{array}{c}\text { Radiação Global } \\
\text { (RG) }\end{array}$ & $\mathrm{Rg}$ & Baseia-se em dados de radiação solar global. \\
\hline
\end{tabular}

*Tmax,Tmin e Tmed: temperaturas diárias máxima, mínima e média, respectivamente; URmed: umidade relativa média diária; Urmin: umidade relativa minima diária; Vv: velocidade do vento; n: fotoperíodo; Pr: pressão atmosférica média diária; Rso: radiação solar; Rstop: saldo de radiação no sistema solo-planta atmosfera; Tpo: Temperatura do ponto orvalho; Pv pressão de saturação de vapor.

Através do programa Microsoft Excel ${ }^{\circledR}$ (2010) foram calculados os valores de ETo pelo modelo de Penman-Monteith-FAO-56 (ETo PMF) e para os demais métodos de estimativas testados, adotando-se uma série histórica contínua de 18 anos de dados climáticos, cujo período base variou de (2000-2017), referentes aos elementos metereológicos específicos de cada método apresentados no quadro 1.

Com o objetivo de estudar o desempenho dos modelos testados em comparação ao modelo padrão (ETo PMF) foi empregado o teste estatístico sugerido por Willmott (1982), que utiliza o índice de concordância "d", que permite observar a dispersão dos valores obtidos por um método qualquer em comparação ao modelo padrão (ETo PMF), Equação (1). Além disso, foram empregados outros índices estatistícos como o coeficiente de Pearson ( $r$ ) e o índice de confiança ou indicador de desempenho, designado pela letra (c), que reúne os coeficientes " $r$ " e "d", conforme as Equações (2) e (3) descritas abaixo.

$\mathrm{d}=1-\left[\frac{\sum\left(\mathrm{P}_{\mathrm{i}}-\mathrm{O}_{\mathrm{i}}\right)^{2}}{\sum\left(\left|\mathrm{p}_{\mathrm{i}}-\mathrm{O}\right|+\left|\mathrm{O}_{\mathrm{i}}-\mathrm{O}\right|\right)^{2}}\right]$

$r=\frac{\sum_{i=1}^{N}\left(\mathrm{O}_{i}-\mathrm{O}\right) \cdot\left(\mathrm{p}_{\mathrm{i}}-\mathrm{P}\right)}{\sqrt{\sum_{\mathrm{i}=1}^{\mathrm{N}}\left(\mathrm{O}_{\mathrm{i}}-\mathrm{O}\right)^{2}} \cdot \sqrt{\sum_{\mathrm{i}=1}^{\mathrm{N}}\left(\mathrm{P}_{\mathrm{i}}-\mathrm{P}\right)^{2}}}$

$c=r \cdot d$

em que,

$\mathrm{Pi}$ - ETo obtida com o modelo avaliado $\left(\mathrm{mm} \cdot \mathrm{d}^{-1}\right)$;

$P$ - Média da ETo do modelo avaliado $\left(m m \cdot \mathrm{d}^{-1}\right)$;

Oi - ETo obtida com o modelo de Penman-Monteith-FAO-56 (ETo PMF) (mm.d-1)

O - Média da ETo do modelo de Penman-Monteith-FAO-56 (ETo PMF) (mm.d ${ }^{-1}$ ).

$O$ índice de confiança ou desempenho varia de 0 quando não há nenhuma concordância entre os modelos a 1,0, quando há perfeita concordância entre os modelos, qualificando o desempenho dos modelos testados segundo a Tabela 1.

TABELA 1- Qualificação do índice desempenho (c), segundo Camargo e Sentelhas (1997).

\begin{tabular}{cc}
\hline $\mathbf{C}$ & Desempenho \\
\hline$>0,90$ & Ótimo \\
0,81 a 0,90 & Muito Bom \\
0,71 a 0,80 & Bom \\
0,51 a 0,70 & Mediano \\
0,41 a 0,50 & Sofrível \\
0,31 a 0,40 & Mau \\
$\leq 0,30$ & Péssimo \\
\hline
\end{tabular}

FONTE: Camargo e Sentelhas,(1997). 
Foram realizadas 6.466 observações em escala diária, compreendendo de janeiro de 2000 à dezembro de 2017. Encontra-se no Gráfico 1 as médias diárias da ETo na escala mensal, obtidas pelos diferentes modelos de estimativa estudados, tendo como padrão o PM-FAO 56.

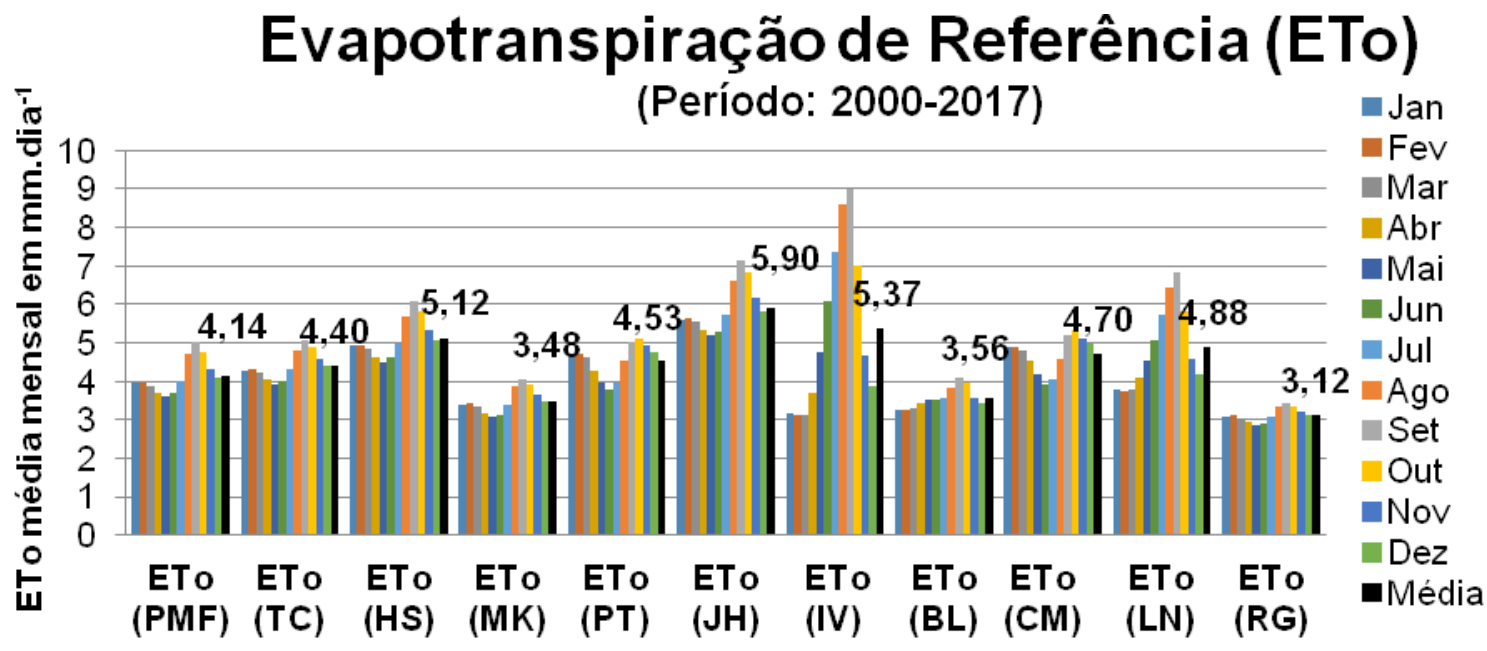

Métodos de estimativa

GRÁFICO 1- Valores médios diários da ETo estimados pelos diferentes métodos.

Para as diferentes equações agrometeorológicas avaliadas para a localidade de Balsas-MA, o valor médio da ETo variou entre 3,12 e 5,90 mm dia ${ }^{-1}$. Como se observa no Gráfico 1, as estimativas de evapotranspiração pelos modelos de Budyko (ETo BK) e Jensen-Haise (ETo JH) tiveram os maiores valores médios diários na escala mensal de ETo, quando comparados aos valores obtidos pelo modelo de Penman-Monteith, cujo valor médio observado foi de $4,14 \mathrm{~mm} \cdot \mathrm{dia}^{-1}$.

As subestimativas e superestimativas apresentadas pelos diferentes modelos avaliados em relação ao modelo de PM-FAO 56 , encontram-se na figura 1 , estas foram obtidas através da razão da diferença entre a evapotranspiração acumulada obtidas pelos diferentes modelos de estimativa e a evapotranspiração acumulada, obtida pela equação de Penman-Monteith-FAO 56, no período estudado. 


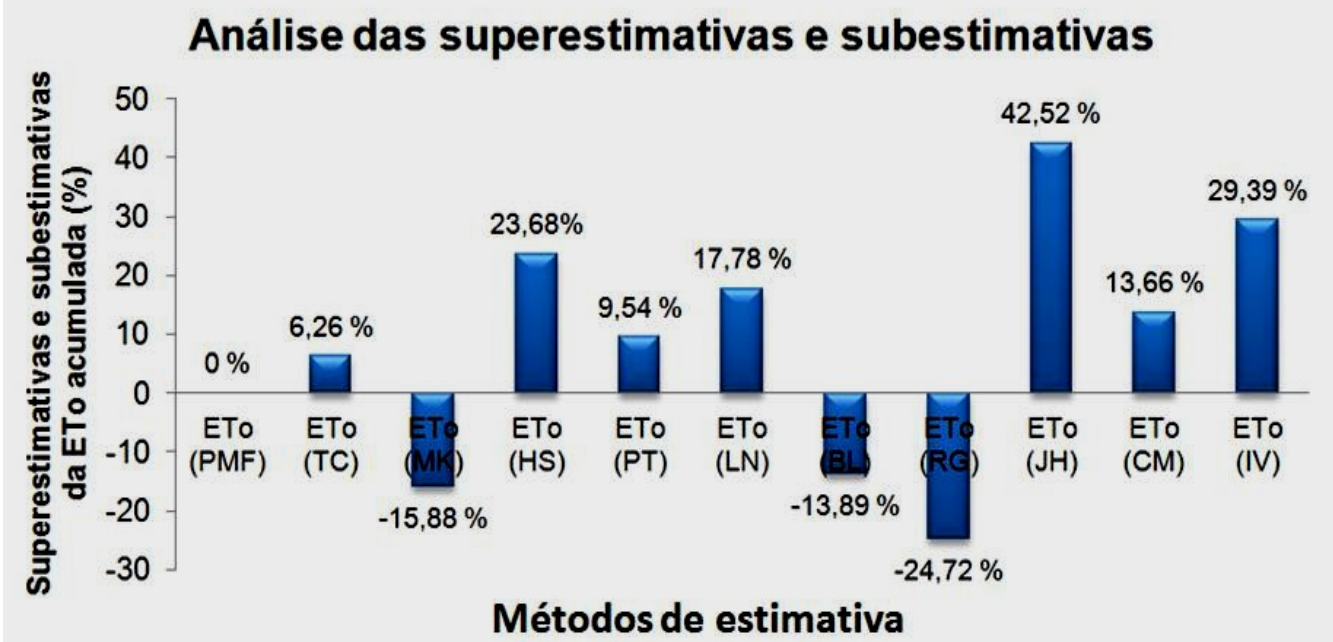

FIGURA 1- Análise de superestimativas e subestimativas dos diferentes modelos em comparação ao modelo padrão de PM-FAO 56.

A equação de Jensen-Haise (ETo $\mathrm{JH})$ superestimou a ETo em todos os meses avaliados, apresentando uma diferença média relativa de $42,52 \%$ em relação a Penman-Monteith-FAO. Resultado semelhante ao obtido neste estudo foi observado no trabalho de Cunha et al. 2013, em que o modelo de Jensen-Haise, superestimou o método padrão em 42,20\%. Jung et al. (2016) avaliando o desempenho do método de Jensen-Haise em uma região do Alto Pantanal de Mato Grosso do Sul, também observaram que este método superestimou a evapotranspiração em 38,8\%. A superestimativa do modelo de Jensen - Haise, provavelmente está relacionada ao fato, que este modelo, foi originalmente desenvolvido para regiões áridas e semi-áridas, assim tende a superestimar a ETo quando utilizado em regiões tropicais de clima quente e úmido.

A equação de Ivanov (ETo IV) superestimou a evapotranspiração de referência em todos os meses avaliados, apresentando diferença média relativa de 29,39 \% em relação ao método padrão de Penman-Monteith-FAO. Essa tendência de superestimativa também foi observada nos estudos de Cunha et al. (2013) em que a superestimativa chegou a $24,41 \%$. Este resultado pode ser atribuído a menor precisão da equação devido a sua simplicidade, pois baseia-se somente na temperatura e umidade relativa do ar para estimar a evapotranspiração.

A equação de Hargreaves - Samani (ETo HG) superestimou a ETo em todos os meses avaliados, com uma diferença média de $23,68 \%$ em comparação ao método padrão. Vasco et al. (2013) estudando a evapotranspiração para a região do município de Rio Real-BA, também observaram a tendência de superestimativa deste método que chegou a $18,88 \%$ em relação à Penman-Monteith. Noia et al. (2014) também verificaram essa tendência de superestimativa do modelo de Hargreaves - Samani em comparação ao método padrão. A equação de Hargreaves - Samani, assim como Jensen-Haise, também foi desenvolvido originalmente para estimar a evapotranspiração em regiões de clima áridos e semi-áridos, logo esperase que este modelo, também, superestime a ETo em climas úmidos.

Como se depreende da análise da Figura 1 a equação de Linacre (ETo LN) superestimou a evapotranspiração em relação à PM-FAO 56 em todos os meses, com uma diferença relativa média de $17,78 \%$. Esta tendência de superestimativa também foi verificada nos estudos de Jung et al. (2016) e Cunha et al. (2013). 
O modelo de Priestley-Taylor (ETo PT) superestimou a evapotranspiração, com uma diferença média relativa de $9,54 \%$. Entretanto, mesmo não foi observado nos estudos de Cunha et al. (2013) e Jung et al. (2016) que observaram tendências de subestimativas da equação de Priestley-Taylor em comparação ao modelo de Penman-Monteith.

A equação que mais se aproximou do modelo de PM-FAO 56 foi o de Turc (ETo TC) que apresentou uma pequena superestimativa da ETo de 6,26 \%. Esta tendência também foi verificada por Cunha et al. (2013), que observaram superestimativa de 10,93 \% do método de Turc em comparação ao modelo padrão.

Já os modelos da Radiação Global (ETo RG), Makkink (ETo MK) e Benevides-Lopez (ETo BL) subestimaram a ETo em 24,72 \%, 15,88\% e 13,89\% , respectivamente.

Os coeficientes de determinacão $\left(R^{2}\right)$ dos métodos estudados para a localidade de Balsas-MA, na escala diária, em relação a ETo PM-FAO 56 apresentaram valores entre 0,90 e 0,62 (Figuras 2 a 6).
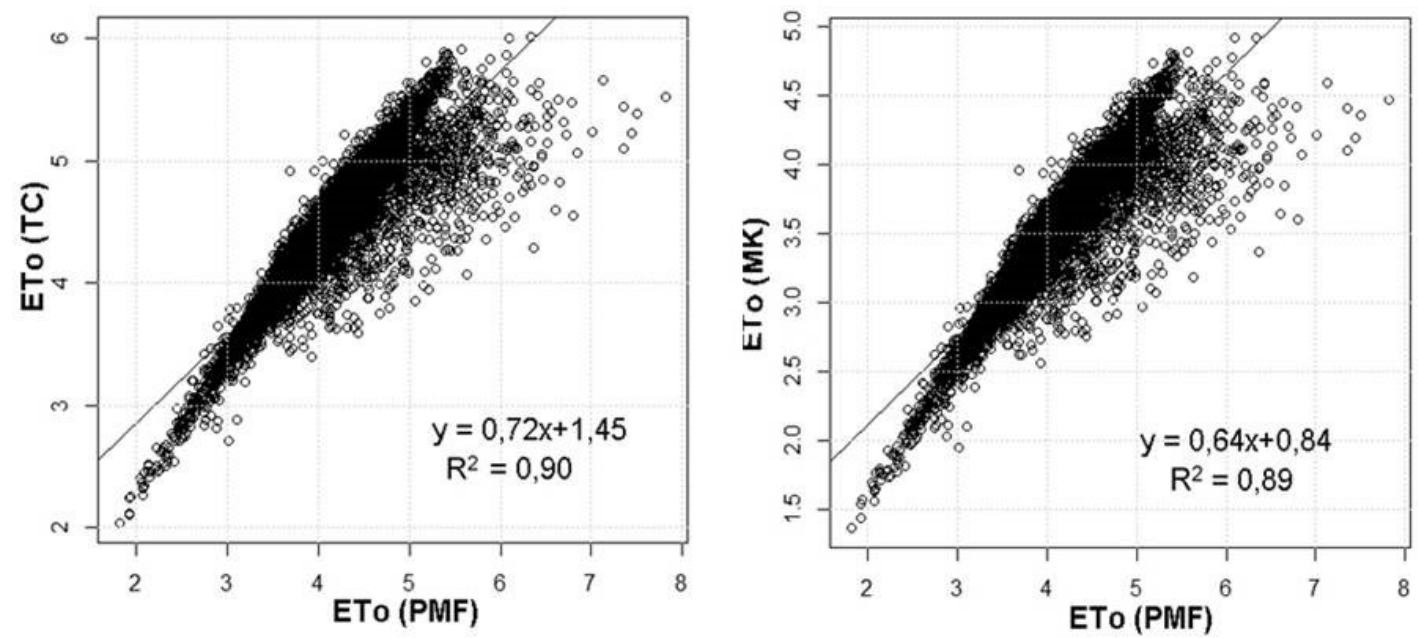

FIGURA 2- Equação de regressão entre os valores de ETo média diária estimadas pelos modelos de Turc (ETo C) e Makkink (ETo MK) em comparação com o modelo de Penman-Monteith-FAO (ETo PMF), Balsas (MA), 2018.
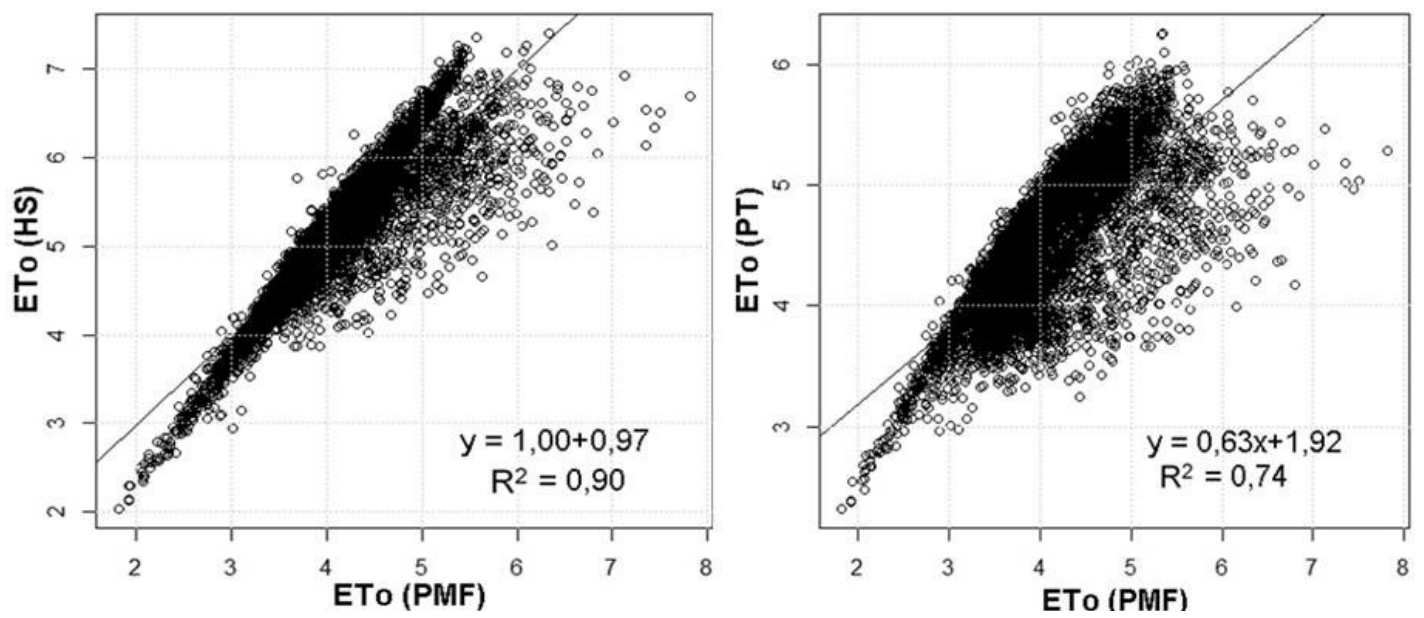

FIGURA 3- Equação de regressão entre os valores de ETo média diária estimadas pelos modelos de Hargreaves \& Samani (ETo HS) e Priestley-Taylor (ETo PT) em comparação com o modelo de Penman-Monteith-FAO (ETo PMF), Balsas (MA), 2018. 

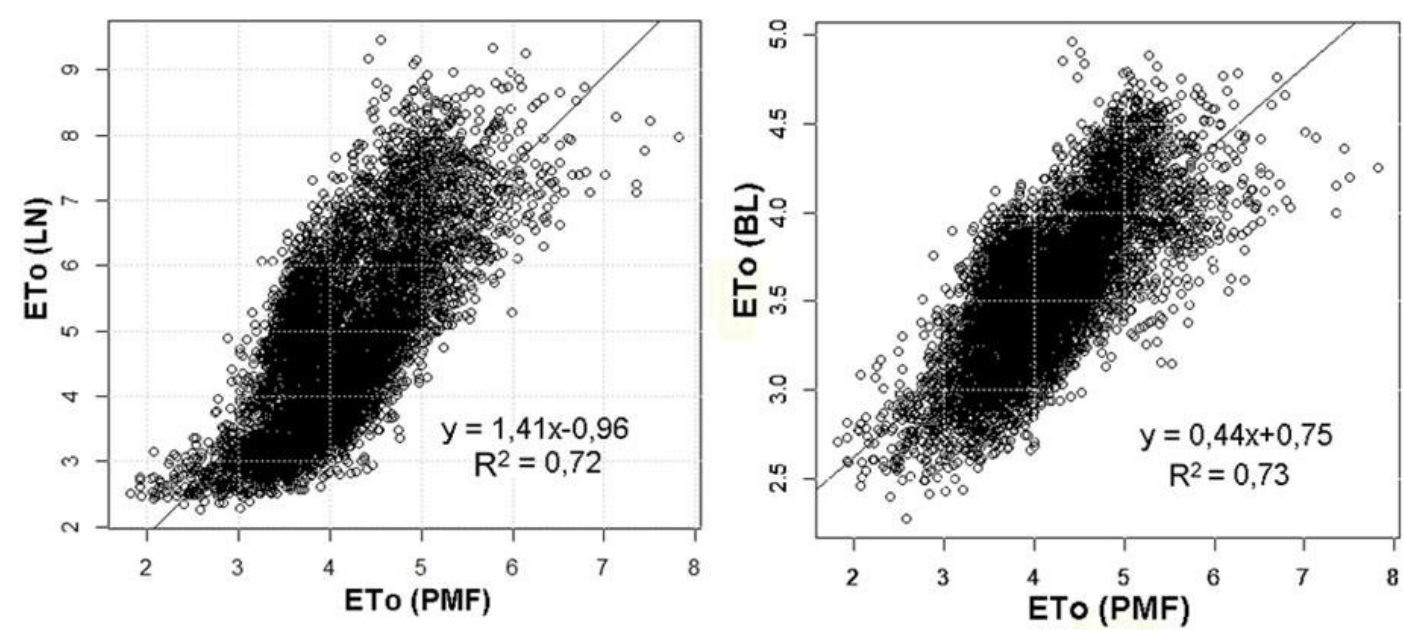

FIGURA 4- Equação de regressão entre os valores de ETo média diária estimadas pelos modelos de Linacre (ETo LN) e Benevides-Lopez (ETo BL) em comparação com o modelo de Penman-Monteith-FAO (ETo PMF), Balsas (MA), 2018.
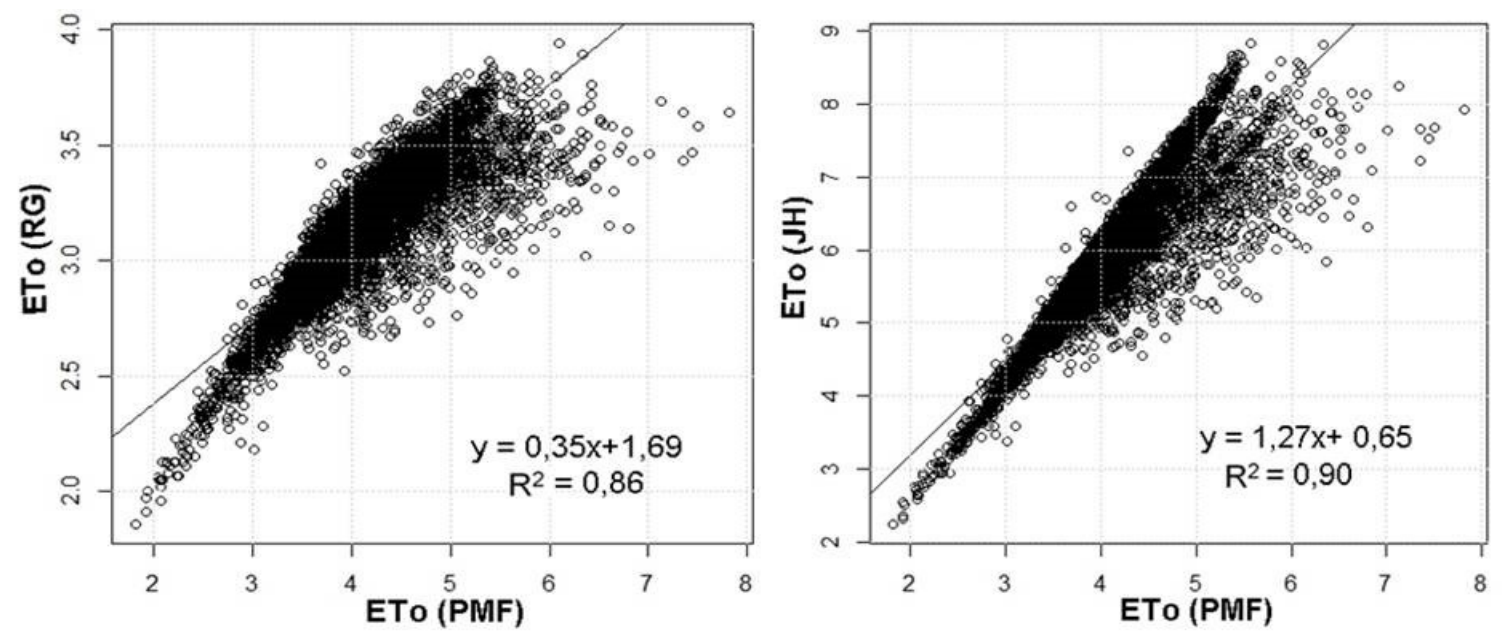

FIGURA 5- Equação de regressão entre os valores de ETo média diária estimadas pelos modelos de Radiação Global (ETo RG) e Jensen-Haise (ETo $\mathrm{JH}$ ) em comparação com o modelo de Penman-Monteith-FAO (ETo PMF), Balsas (MA), 2018. 

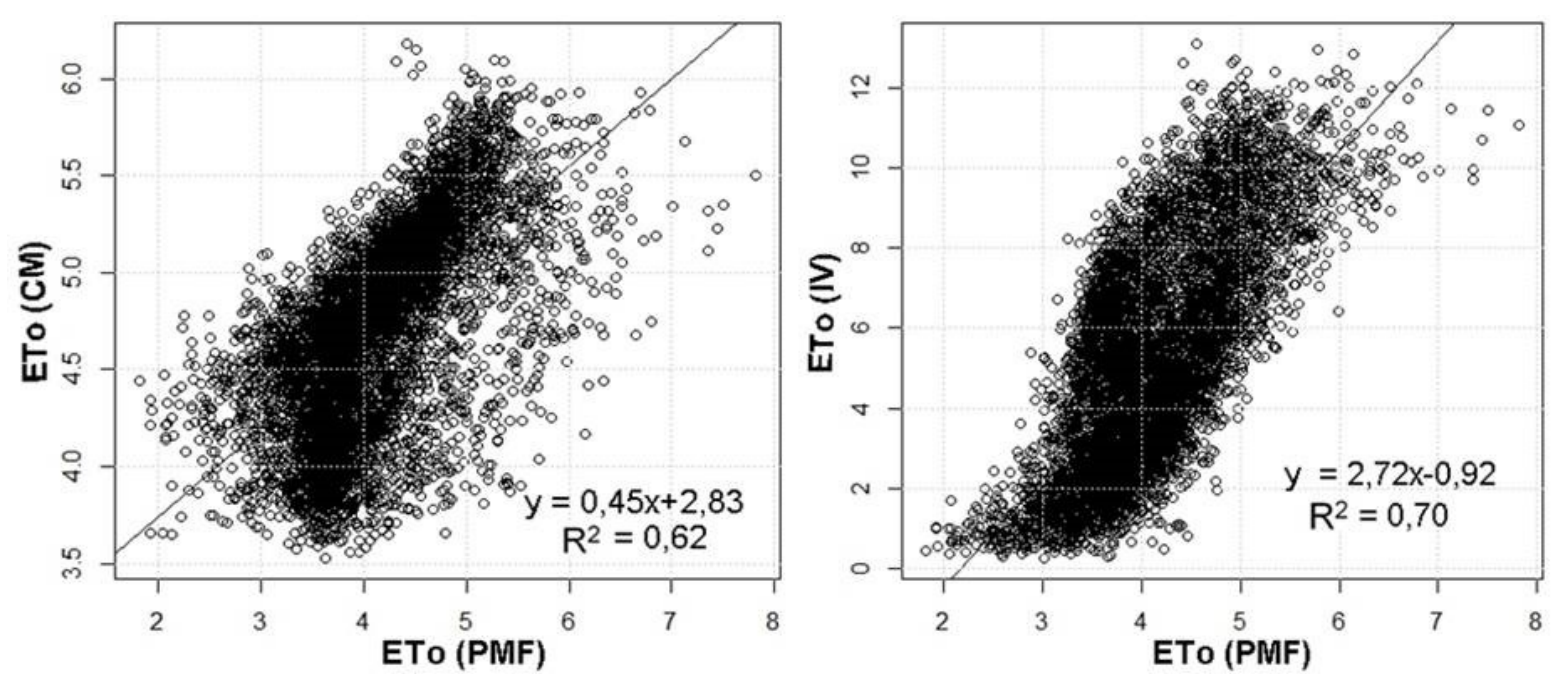

FIGURA 6- Equação de regressão entre os valores de ETo média diária estimadas pelos modelos de Camargo (ETo CM) e Ivanov (ETo IV) em comparação com o modelo de Penman-Monteith-FAO (ETo PMF), Balsas (MA), 2018.

Observa-se nas análises das Figuras 2 a 6 que as equações empíricas que apresentaram melhor ajuste em relação à Penman-Monteith-FAO, na escala diária, com o coeficiente de determinação $\left(R^{2}\right)$, foram os modelos de Turc $\left(R^{2}=0,90\right)$, Hargreaves - Samani $\left(R^{2}=0,90\right)$, Jensen-Haise $\left(R^{2}=0,90\right)$ e Makkink $\left(R^{2}=0,89\right)$, constata-se que os dois modelos baseados na radiação, Turc e Makkink, e os dois modelos desenvolvidos para condições áridas e semi-áridas, Hargreaves \& Samani e Jensen-Haise, como o observado apresentaram ajustes satisfatórios com 0 modelo padrão nas condições climáticas de Balsas-MA. Entretanto, segundo Barros et al. (2009), a utilização do coeficiente de determinacão $\left(R^{2}\right)$ como o único indicador de performance de um determinado modelo não é adequado, pois este índice não leva em consideração o tipo e a magnitude das discrepâncias entre o valor observado com o padrão e o obtido com o modelo testado.

Já os piores ajustes dos modelos testados do coeficiente de determinação da ETo $\left(R^{2}\right)$, em comparação com o método padrão foram: Camargo $\left(R^{2}=0,62\right)$, Ivanov $\left(R^{2}=0,70\right)$, Linacre $\left(R^{2}=0,72\right)$ e Benevides-Lopez $\left(R^{2}=0,73\right)$. Esses ajustes, obtidos com estes modelos, podem atribuidos à simplicidade de suas equações que conferem menor precisão ao método, pois a maioria apresentam um número reduzido de parâmetros de entrada na estimativa da evapotranspiração.

A Tabela 3, mostra os valores do coeficiente de correlação "r" e de Willmot "d" e o índice de confiança "c" dos modelos testados, na escala diária, a localidade de Balsas, Maranhão.

TABELA 3- Coeficiente de correlação "r", "d" de Willmott e desempenho "c" dos modelos avaliados, na escala diária.

\begin{tabular}{cccccc}
\hline MÉTODOS & $\%$ & "d" & "r" & "c" & DESEMPENHO \\
\hline AVALIAÇÃO DO DESEMPENHO NA ESCALA & MÉDIA DIÁRIA \\
\hline Penman-Monteith-FAO 56 (PMF) & 100,00 & 1 & 1 & 1 & Ótimo \\
Turc (TC) & 106,26 & 0,89 & 0,89 & 0,80 & Bom \\
Makkink (MK) & 84,12 & 0,73 & 0,89 & 0,65 & Mediano \\
Hargreaves e Samani (HS) & 123,68 & 0,68 & 0,90 & 0,61 & Mediano \\
Priestley-Taylor (PT) & 109,54 & 0,78 & 0,74 & 0,58 & Mediano
\end{tabular}

ENCICLOPÉDIA BIOSFERA, Centro Científico Conhecer - Goiânia, v.15 n.27; p. 9 


\begin{tabular}{cccccc} 
Linacre (LN) & 117,78 & 0,67 & 0,72 & 0,49 & Sofrível \\
Benevides-Lopez (BL) & 86,11 & 0,66 & 0,73 & 0,49 & Sofrível \\
Radiação Global (RG) & 75,28 & 0,54 & 0,86 & 0,46 & Sofrível \\
Jensen-Haise (JH) & 142,52 & 0,51 & 0,90 & 0,45 & Sofrível \\
Camargo (CM) & 113,66 & 0,66 & 0,62 & 0,41 & Sofrível \\
Ivanov (IV) & 129,39 & 0,48 & 0,70 & 0,33 & Mau \\
\hline
\end{tabular}

A análise da Tabela 3 permitiu verificar que dentre os dez modelos de estimativa da ETo estudados, o que mostrou melhor desempenho na escala diária em relação à Penman-Monteith FAO-56 foi o método de Turc (ETo TC), apresentado desempenho "Bom" na escala diária. Em seguida, tem-se o método de Makkink (ETo MK), com desempenho "Mediano" na escala escala diária, com coeficiente de correlação 0,89 e índice de confiança de 0,65. O desempenho satisfatório do modelo de Turc (ETo TC) também constatado nos estudos de Cunha et al. (2013) para a localidade de Chapadão do Sul, MS. E Morais et al. (2016), também obtiveram desempenho mediano do método de Makkink para a localidade de Bebedouro, PE. Os modelos de Turc e Makkink são equações empirícas baseadas na radiação e geralmente essas equações exibem melhor desempenho em comparação aos modelos baseados somente na temperatura do ar Moura et al. (2013).

Os modelos de Hargreaves - Samani e Priestley-Taylor ambos apresentaram desempenho "Mediano" na escala diária. Já no estudo realizado por Passos et al. (2017), os modelos de Priestley-Taylor e Hargreaves - Samani receberam a classificação de "Bom" e "Ruim", respectivamente, para as condições climáticas do município de Chapadinha, Estado do Maranhão. É provável que esta divergência esteja relacionada a quantidade de observações, pois no estudo de Passos et al. 2017, foram utilizados dados climáticos de apenas um ano para a análise de desempenho o dos modelos.

Lucena et al. (2016), em seus estudos realizados em Bom Jesus-PI, obtiveram desempenho "Sofrível" para o método de Hargreaves - Samani, enquanto Paiva e Souza (2016) estudando a evapotranspiração de referência em Piraí do SulPR, obtiveram desempenho "Ruim" e " $r$ " igual a 0,23 para o mesmo modelo. Nota-se que estes autores não encontraram ajustes satisfatórios na utilização da equação de Hargreaves - Samani o que pode ser atribuído ao fato desta equação ter sido desenvolvida para regiões áridas, diferente das regiões estudadas pelos referidos autores. Diferente dos resultados encontrados nesse trabalho Cunha et al. (2013), obtiveram um desempenho "Sofrível" nos testes do modelo de Priestley-Taylor em Chapadão do Sul, MS e atribuíram este resultado ao fato deste modelo desconsiderar a radiação e a velocidade do vento na obtenção da evapotranspiração.

Os modelos de Linacre $(c=0,49)$, Benevides-Lopez $(c=0,49)$, Radiação Global $(c=0,46)$, Jensen-Haise $(c=0,45)$ e Camargo $(c=0,41)$ apresentaram desempenho sofrível. Em seus estudos Jung et al. 2016, também obtiveram um desempenho "Sofrível" no cálculo da ETo pelo método de Linacre, tanto no período seco quanto chuvoso, para regiões do Alto Pantanal, MS. Já Rocha et al. 2015 obtiveram desempenho "Péssimo" no cálculo da ETo por Linacre para a localidade de Garanhuns, PE. Jung et al. 2016 também obtiveram desempenho "Sofrível" ao avaliar a ETo pelo o modelo de Benevides-Lopez no Alto Pantanal-MS, desaconselhando o uso para estimativa da ETo nessa região. Já Cunha et al. (2013) 
obtiveram desempenho "Mediano" na estimativa da ETo utilizando o método da Radiação Global para a região de Chapadão do Sul, MS.

No estudo realizado por Passos et al. (2017), a equação de Jensen-Haise, também não apresentou desempenho satisfatório, para a localidade de Chapadinha, Estado do Maranhão, e foi categorizado como "Ruim". Entretanto, diferente dos resultados obtidos nesse estudo Morais et al. (2016), encontraram um desempenho "Bom" para a equação de Jensen-Haise em Petrolina-PE e Silva et al. (2014), constataram um desempenho "Mediano" para a equação de Jensen-Haise em Fernando de Noronha, PE.

Semelhante aos resultados obtidos nesse estudo a avaliação da equação de Camargo por Moura et al. 2013 apresentou desempenho "Sofrível", na escala diária, para a região de Vitória de Santo Antão, Estado de Pernambuco. Já Passos et al. (2017) obtiveram a classificação "Péssimo" na obtenção da ETo pelo método de Camargo para a localidade de Chapadinha, Maranhão.

A equação de Ivanov (ETo IV) apresentou o pior ajuste entre os modelos avaliados sendo classificado como "Mal", na escala diária, com coeficiente de correlação 0,70 e índice de confiança de 0,33. No estudo realizado por Cunha et al. 2013, o modelo de Ivanov também apresentou desempenho "Mal", no estudo da ETo em Chapadão do Sul, MS. Ribeiro et al. (2015) também não obtiveram resultados satisfatórios na avaliação da ETo pelo método de Ivanov para a localidade de Sobral, CE.

A maior parte dos modelos testados apresentaram valores reduzidos para o índice "c", evidenciando os desempenhos insatisfatórios em comparação ao método padrão (ETo PMF), destacando o ajuste apresentado pelo modelo de Turc (ETo TC) com $c=0,80$ e $r=0,89$. Já os modelos de Linacre (ETo LN) Benevides-Lopez (ETo $B L)$, Radiação Global (ETo RG), Jensen-Haise (ETo JH), Camargo (ETo CM) e Ivanov (ETo IV) não são recomendados para estimativa da ETo para a região do município de Balsas/MA.

\section{CONCLUSÕES}

1. Dentre os modelos testados, a equação de Turc (ETo TC) apresentou melhor índice de confiança 0,80 "Bom", apresentando maior confiabilidade na avaliação da evapotranspiração de referência.

2. Os modelos de Linacre, Benevides-Lopez, Radiação Global, Jensen-Haise, Camargo e Ivanov apresentaram os piores desempenhos em comparação ao método de Penman-Monteith-FAO, não apresentando a precisão necessária para a utilização prática na obtenção da evapotranspiração de referência para o município de Balsas/MA.

\section{REFERÊNCIAS}

BARROS, V.R.; SOUZA, A.P.; FONSECA, D.C.; SILVA, L.B.D. Avaliação da evapotranspiração de referência na região de Seropédica, Rio de Janeiro, utilizando lisímetro de pesagem e modelos matemáticos. Revista Brasileira de Ciências Agrárias, Recife, v.4, n.2, p.198-203, 2009. Disponível em: < http://www.agraria.pro.br/ojs- 2.4.6/index.php? journal=agraria\&page=article\&op=view\&path $\% 5 B \% 5 D=103>$. doi:10.5039/agraria.v4i2a13 
CARVALHO, D. F.; DA ROCHA, H. S.; BONOMO, R. \& DE SOUZA, A. P. Estimativa da evapotranspiração de referência a partir de dados meteorológicos limitados. Pesquisa Agropecuária Brasileira, v. 50, n. 1, p. 1-11, 2015. Disponível em: < http://seer.sct.embrapa.br/index.php/pab/article/view/19629>. doi: DOI: 10.1590/S0100-204X2015000100001

CUNHA, F. F.; MAGALHÃES, F. F.; CASTRO, M. A. Métodos para estimativa da evapotranspiração de referência para Chapadão do Sul - MS. Engenharia na agricultura, Viçosa - MG, v.21 n.2, mar/abr. 2013. Disponível em: < http://www.seer.ufv.br/seer/index.php/reveng/article/view/346>. doi: https://doi.org/10.13083/reveng.v21i2.346.

FERREIRA, A. O.; RESENDE, J. C.; ARAÚJO, M. C. A. Plano de Capacitação profissional para a região do Matopiba: Estudo de Viabilidade. AGRO EM QUESTÃO: Revista de Iniciação Científica da Faculdade CNA, p. 45, 2017. Disponível em: < http://faculdadecna.com.br/media/download/RevistaCNA61708.1.pdf\#page=47>.

GIOVANELLI, L. B.; DE OLIVEIRA, R. A.; DA COSTA OLIVEIRA FILHO, J.; SEDIYAMA, G. C.; CECON, P. R \& BAPTESTINI, J. C. M. Influência de Elementos Meteorológicos na Evapotranspiração estimada pelo irrigâmetro. IRRIGA, v. 21, n. 1, p. 58-73, 2016. Disponível em: < http://irriga.fca.unesp.br/index.php/irriga/article/view/1141>. doi: http://dx.doi.org/10.15809/irriga.2016v21n1p58-73

JUNG, L. H.; BISCARO, G. A.; OLIVEIRA, G. Q.; ALVES, M. A.; GIACON, G. M. Estimativa da evapotranspiração de referência em uma região do Alto Pantanal. Magistra, Cruz das Almas - BA, V. 28, N.2, p.168-177, Abri/Jun.2016. Disponível em: <https://magistraonline.ufrb.edu.br/index.php/magistra/article/view/265>.

LUCENA, F.A.P.; SIVA, E.M.; RIBEIRO, A.A.; SIMEÃO, M.; LUCENA, J.P.P. Comparação entre métodos de estimativa da evapotranspiração de referência no município de Bom Jesus, PI. Revista Brasileira de Agricultura Irrigada, v.10, n.3, p. 663-675, 2016. Disponível em: http://www.inovagri.org.br/revista/index.php/rbai/article/view/404/pdf_282>. doi: 10.7127/rbai.v10n300404

MORAIS, J. E. F.; SILVA,T. G. F.; SOUZA, L. S. B.; MOURA,M. S. B.; DINIZ, W. J. S.; SOUZA, C. A. A. Avaliação do método de Penman Monteith FAO 56 com dados faltosos e de métodos alternativos na estimativa da evapotranspiração de referência no Submédio Vale do São Francisco. Revista Brasileira de Geografia Física, v. 8, n. $6, \quad$ p. 1644-1660, 2016. Disponível em: < https://periodicos.ufpe.br/revistas/rbgfe/article/view/233665>.

MOURA, A.R.C.; MONTENEGRO, S.M.G.L.; ANTONINO, A.C.D.; AZEVEDO, J.R.G.; SILVA, B.B.; OLIVEIRA, L.M.M. Evapotranspiração de referência baseada em métodos empíricos em bacia experimental no estado de Pernambuco - BRASIL. Revista Brasileira de Meteorologia, v.28, n.2, 181 - 191, 2013. Disponível em: < http://www.ingentaconnect.com/content/doaj/01027786/2013/00000028/00000002/art 00007>. doi: https://doi.org/10.1590/S0102-77862013000200007. 
NOIA, C. P. Z.; PEREIRA, S. B.; ROSA, D. R. Q; ALMEIDA, R. V. Evapotranspiração de referência estimada pelos métodos Penman-Monteith-FAO (56) e Hargreaves \& Samani para o município de Dourados, MS. Revista Agrarian, Dourados, v.7, n.24, p.300-308, 2014. Disponível em: < http://ojs.ufgd.edu.br/index.php/agrarian/article/view/2476>. https://doi.org/10.30612/agrarian.v7i24.2476.

PALARETTI, L. F.; MANTOVANI, E. C.; SEDIYAMA, G. C. Análise da sensibilidade dos componentes da equação de Hargreaves-Samani para a região de BebedouroSP. Revista Brasileira de Meteorologia. Sociedade Brasileira de Meteorologia, v. 29, n. 2, p. 299-306, 2014. Disponível em: < http://200.145.6.238/handle/11449/109952>. doi: http://dx.doi.org/10.1590/S010277862014000200012

PAIVA, C. M \& DE SOUZA, A. D. S. P. Avaliação de Métodos de Estimativa da Evapotranspiração de Referência para Fins de Manejo da Irrigação. Anuário do Instituto de Geociências, v. 39, n. 1, p. 42-51, 2016. Disponível em: < http://www.ppegeo.igc.usp.br/index.php/anigeo/article/view/8476>.

doi: http://dx.doi.org/10.11137/2016_1_42_51

PASSOS, M. L.V.; RAPOSO, A. B.; MENDES, T. J. Evapotranspiração de referência por diferentes métodos para o município de Chapadinha-MA. Brazilian Journal of Applied Technology for Agricultural Science, Guarapuava-PR, v.10, n.1, p.59-66, $2017 . \quad$ Disponível em: https://revistas.unicentro.br/index.php/repaa/article/view/4745>. doi: 10.5935/PAeT.V10.N1.06.

RIBEIRO, A. A.; SIMEÃO, M.; SOARES, J. M.; MOURA, R. S. Avaliação de modelos de estimativa da evapotranspiração de referência em Sobral, CE. Revista Agrogeoambiental, Pouso Alegre, v. 7, n. 4, p. 71-81, dez. 2015. Disponível em: < https://agrogeoambiental.ifsuldeminas.edu.br/index.php/Agrogeoambiental/article/vie w/660>. doi: http://dx.doi.org/10.18406/2316-1817v7n42015660.

ROCHA, I. P.; DA SILVA LIMA, N.; CHAGAS, R. M \& DE ALMEIDA, G. L. P. Comparação entre equações empíricas para estimativa da evapotranspiração de referência para o município de Garanhuns, PE| Comparison of empirical equations to estimate the reference evapotranspiration for the city of Garanhuns, PE. Revista Geama, v. $1, \quad$ n. 2, $\quad$ p. 112-127, 2015. Disponível em: < http://www.ead.codai.ufrpe.br/index.php/geama/article/view/487>

RODRIGUES, R. R.; PIZETTA, S. C.; REIS, E. F.; RIBEIRO, W. R.; CAPELINI, V. A. Estimativa da demanda hídrica do cafeeiro conilon, variedade Robusta tropical, em comparação com a evapotranspiração obtida pelo método de Hargreaves \& Samani. Enciclopédia biosfera, Centro Científico Conhecer - Goiânia, v.9, n.16; p. 1055. 2013. Disponível em: http://www.conhecer.org.br/enciclop/2013a/agrarias/ESTIMATIVA\%20DA $\% 20 D E M A N D A . p d f>$. 
SILVA, J.L.R; MONTENEGRO, A. A. A.; SANTOS, T. E.M.; SANTOS, E.S. Desempenho de diferentes métodos de estimativa da evapotranspiração de referência para Fernando de Noronha. Irriga, Botucatu, v. 19, n. 3, p. 390-404, julset., 2014. Disponível em: < http://200.145.140.50/index.php/irriga/article/view/658>. doi: http://dx.doi.org/10.15809/irriga.2014v19n3p390.

VASCO, A. N.; NETTO, A. D. O. A.; FACCIOLI, G. G.; CHAGAS, R. M.; DE SOUSA, I. F \& DA SILVA, M. G. Comparação entre métodos de estimativa da Evapotranspiração de referência (eto) no município de Rio Real-BA. Irriga, Botucatu, v. 18, n. 1, p. 351-363, abril-junho, 2013.

Disponível em: < http://irriga.fca.unesp.br/index.php/irriga/article/view/558>. doi: DOI: https://doi.org/10.13083/reveng.v23i1.516. 